\title{
Mediation vs pseudomediation: An unresolved issue '
}

WILLIAM A, HAYES

GEORGE PEABODY COLLEGE FOR TEACHERS

Previous studies related to the mediation-pseudomediation controversy have questioned either the replicability or the assumptions underlying the pseudomediation effect. It is suggested that a more profitable line of research would be to determine the factors that contribute to the pseudomediation effect. Intertrial interval may be critical.

The mediation vs pseudomediation controversy was initiated by Mandler \& Earhard's (1964) assertion that mediational effects found in some verbal learning studies may only be apparent. They contend that in a typical chaining paradigm ( $A-B, B-C, A-C)$ the learning of the second stage produces unlearning or forgetting of the first stage, whereas in the control paradigm (A-B, D-C, $A-C)$ no such process operates. Consequently, there is little interference in the final stage A-C learning of the chaining paradigm, but there is an appreciable amount in the control paradigm, thus producing apparent mediation. They supported their argument with a pseudomediation experiment which compared learning of an $A-B, B-C, A-E$ (Pseudomediation) paradigm with an $A-B, D-C, A-E$ (Control) paradigm. The A-E learning of the first paradigm was facilitated relative to the A-E learning of the second paradigm without opportunity for mediation.

Jenkins \& Foss (1965) attempted to replicate the pseudomediation effect and test the unlearning assumption by assessing the recall of $A-B$ pairs after the second and third stages for both pseudomediation and control groups. Their results showed that recall of first list responses following third list learning did not differ from E and C groups. Similarly Goulet (1966) found potential mediators to be at least as available in paradigms conducive to producing mediation as the analogous terms in the control paradigms.

Earhard \& Mandler (1965) used an association task to test for the availability of Stage $I$ associations. Of the paradigms used (A-B, D-C; $A-B, C-A$; and $A-B$, $C-D)$ the lowest mean number of associations were with the A-B, B-C paradigm, while the A-B, C-D paradigm had the highest.

Schulz, Weaver, \& Ginsberg (1965) used pseudomediation and mediation paradigms and their controls in an attempted replication of Mandler \& Earhard's effect. They showed a clear mediation effect while failing to show a pseudomediation effect. Similar results were reported by Goulet \& Postman (1966) using methodology that more closely approximated that of Mandler \& Earhard.

Carlson (1966), like Schulz et al, and Goulet \&
Postman used both mediation and pseudomediation paradigms and their controls in an attempt to replicate Mandler \& Earhard's results. Carlson obtained the pseudomediation effect but not the mediation effect. In addition, he used both the recall and the association tasks in testing for the avallability of the B items following Stage II learning. Neither recall nor matching produced differences between groups of A-B unlearning.

Selm \& Penny (1966) used a paired association task with pictures as stimuli to test three predictions: (1) Stage II learning by the control pairs would be superior to that of the experimental pairs; (2) Stage III learning of experimental pairs would be superior to that of the control pairs; and (3) fewer $B$ responses would be available to the Stage III stimuli of the experimental pairs than to the control pairs as measured by an association task after Stage II learning. The first two predictions were confirmed, but the association data failed to support the third.

Goulet \& Postman noted that all of the above studies have sought to answer one or more of the following questions: (a) Is the unlearning assumption tenable? (b) Can the pseudomediation effect be replicated? (c) Can the pseudomediation effect and the mediation effect be shown to occur together? While these studies provide data to answer these questions, a fourth and extremely important question has not been asked; namely, what are the factors peculiar to the studies that demonstrated pseudomediation, i.e., why was the pseudomediation effect obtained? Any resolution of the controversy that is to be considered adequate must provide an answer to this last question.

\section{Unlearning Assumption}

The unlearning assumption leads to the prediction that given an $A-B, B-C$ sequence and its control $A-B$, D-C, learning in Stage II should be faster for the control group. The testing of this prediction provides an indirect means of testing the unlearning assumption. A more direct method involves the direct testing of the availability of the B items following Stage II learning. As previously indicated, two different procedures have been used for testing for the avallability of B terms. Jenkins \& Foss (1965) used a recall task while Earhard \& Mandler (1965) used an association task.

Virtually all of the studies found that Stage II learning was faster for the controls than for the experimental Ss, thus providing partial support for the unlearning assumption. On the other hand, of the five studies that tested directly for the avallability of the B terms (Jenkins \& Foss, 1965; Earhard \& Mandler, 1965; 
Carlson, 1966; Goulet, 1966; Seim \& Penny, 1966) only Earhard and Mandler's study provided support for the unlearning assumption. It should also be noted that Carison used both recall and free-association tests of the availability of $B$, but failed to find differences between experimental and control groups on either measure while demonstrating the pseudomediation effect. The unlearning assumption is further weakened by the fact that two studies which showed that Stage II learning was superior for controls also demonstrated that the availability of $B$ did not differ for experimental and control groups (Jenkins \& Foss; Seim \& Penny). Thus, it appears that negative transfer evidenced in Stage II learning is independent of the availability of $B$ following Stage II learning, and since it is the latter that would produce interference it may be concluded that the unlearning hypothesis is not supported by the available data.

\section{Replicability of Pseudomediafion}

The major problem with most attempts to replicate the pseudomediation effect (Jenkins \& Foss; Schulz et al; Goulet \& Postman) appears to lie in their failure to replicate Mandler and Earhard's procedures. Carlson's study which most closely replicated Mandler and Earhard's procedures did obtain the pseudomediation effect. Therefore, one is forced to conclude that the pseudomediation effect is replicable, but is closely related to the experimental procedures used.

\section{Joint Occurrence of Mediation and Pseudomediation}

Of the studies that used mediation and pseudomediation paradigms in the same experiment (Schulz et al; Goulet \& Postman; Carlson; Seim \& Penny) only Seim \& Penny found both mediation and pseudomediation. It should be noted, however, that Seim \& Penny used a paired association task with pictures of the PPVT as stimuli. The conclusion, then, is that mediation and pseudomediation have not been demonstrated to occur in the same verbal learning experiment.

\section{Suggested Resolution}

Perhaps the most obvious conclusion that can be drawn from the present review is that the pseudomediation effect is real. The remaining task is to answer the fourth question. A comparison of the methodology used in the various studies suggests that the intertrial interval may be a critical variable, as Carlson has suggested. Of the studies reporting the pseudomediation effect (Mandler \& Earhard; Carlson) both used no intertrial interval, a methodological difference that distinguishes them from all the other studies. Further approaches to the controversy should take the form of manipulating the intertrial interval across a continuum for both mediation and pseudomediation paradigms and assessing their effects. It is conceivable that the probability of demonstrating mediation is greatest when the intertrial interval is above some critical limit, whereas the probability of obtaining the pseudomediation effect is maximized by the use of a small or no intertrial interval. This position is entirely consistent with the available data and provides clear answers to the four major experimental questions. First, since this position postulates a methodological difference as the basis for the distinction between mediation and pseudomediation, the unlearning assumption is irrelevant. No attempt is made to specify the underlying processes (unlearning, less time for rehearsal, etc.) operating to produce the different phenomena. Secondly, the failure of all attempted replications of Mandler and Earhard's effect may be attributed to the use of either 4 or $8 \mathrm{sec}$ intertrial intervals. Thirdly, the failure to demonstrate the occurrence of mediation and pseudomediation in the same verbal learning experiment may be attributed to the fact that all such attempts have used either no intertrial interval for both mediation and pseudomediation paradigms or a 4 or $8 \mathrm{sec}$ intertrial interval for both paradigms.

Mediation is not an artifact, therefore pseudomediation cannot be posited as a phenomenon that represents a better explanation of the mediation phenomenon. Rather, it represents a different and mutually exclusive phenomenon functionally related to a variation in the procedures used to demonstrate mediation.

\section{References}

Carlson, J. G. Pseudomediational effects with no intertrial intervals. Psychon. Sci., 1966, 5, 77-78.

Earhard, B., \& Mandler, G. Pseudomediation: A reply and more data. Psychon. Sci., 1965, 137-138.

Goulet, L. R. Retroaction and the "fate" of the mediator in three stage mediation paradigms. J. verbal Learn. verbal Behav., $1966,5,172-176$.

Goulet, L. R., \& Postman, L. An experimental evaluation of the pseudomediation hypothesis. Psychon. Sci., 1966, 4, 163-164.

Jenkins, J. J. Comments on pseudomediation. Psychon. Sci., $1965,2,97-98$.

Jenkins, J. J., \& Foss, D. An experimental analysis of pseudomediation. Psychon. Sci., 1965, 2, 99-100.

Mandler, G., \& Earhard, B. Pseudomediation: Is chaining an artifact? Psychon. Sci., 1964, 1, 247-248.

Schulz, R., Weaver, G., \& Ginsberg, S. Mediation with pseudomediation controlled: Chaining is not an artifact! Psychon. Sci., 1965, 2, 169-170.

Seim, R. D., \& Penny, R. K. A free-association test of the diffeential unlearning hypothesis of pseudomediation. Psychon. Sci., $1966,5,143-144$.

\section{Note}

1. The author's research is supported in part by NICHD grant HD-43. 